\title{
PARAMETERS OF OPTIMUM HIERARCHY STRUCTURE IN AHP
}

\author{
Stan Lipovetsky \\ GfK Custom Research North America \\ Mineapolis, MN, USA \\ E-mail: stan.lipovetsky@gfk.edu
}

\begin{abstract}
A problem of finding optimal hierarchy parameters for a given number of alternatives is considered. Hierarchical structures are widely used in the Analytic Hierarchy Process, Conjoint Analysis, and various other methods of Multiple Criteria Decision Making. The suggested approach is based on minimizing the objective of total pair comparisons across all the hierarchy structure. For an optimal hierarchy, the minimum effort is needed for eliciting data and synthesizing the local preferences across the hierarchy to get the global priorities or utilities. The obtained analytical and numerical results show how to choose the optimal structuring of the alternatives into groups by sub-criteria, criteria, and hyper-criteria by many-level hierarchy. The obtained results are beneficial for practical managerial decision making in the complex problems with numerous alternatives.
\end{abstract}

Keywords: AHP, Hierarchy Optimization, Pair Comparisons.

\section{Introduction}

Designing a hierarchy usually presents the first task in the AHP or other approaches to multiattribute decision making. A hierarchy configuration can be outlined by scrutiny of the connections among the alternatives for combining them into groups of different criteria. Then all pair comparisons data can be elicited from an expert for each level of the hierarchy, thus, among the items by each criterion, and among the criteria themselves. Statistical design for reducing the number of pair comparisons is applied in the case of many respondents, especially, in the conjoint analysis. The paper considers a problem of assembling a hierarchy structure in the optimizing approach of finding the minimum number of needed pair comparisons among the alternatives, subcriteria, and criteria. In many situations a researcher does not have prior information on a possible hierarchical design, so using the results of the optimization technique can help to facilitate the data eliciting process for local and global priorities. More specifically, for a given number $n$ of all the alternatives, it is possible to estimate how many of them should be combined into each group of the lower level, and how many these groups of the criteria are needed at the upper levels so that the total number of all the required pair comparisons across the hierarchy structure reaches its minimum. This approach can serve to various practical aims of managerial decision makers.

\section{Literature Review}


ISAHP Article: Lipovetsky, Paper Proposal Submitted to the International Symposium of the Analytic Hierarchy Process 2014, Washington D.C., U.S.A.

Researchers working with AHP methodology and applications should be familiar with Saaty's classical monographs $(1980,1996)$, and others. But what is less known, T. Saaty is the author of the monograph (1970) on the optimization in integers, for instance, developed and widely applied in linear and goal programming and various other operation research algorithms. The current work is motivated by the ideas in Saaty (1970), although uses not the complex techniques of integer optimization but only a general setup and approximate solutions of finding integer solutions by the rounded continuous optimal values of the hierarchical structures. Applications of these results have been used in solving practical complex problems in marketing research (Lipovetsky, 2006, 2009).

\section{Hypotheses/Objectives}

The main aim of this study consists in an advance evaluating of a set of appropriate parameters of a hierarchy sizes which then can be used in the time-money-efforts-saving AHP data eliciting process because it would need finding the minimum number of all the pair comparisons across all the hierarchy.

\section{Research Design/Methodology}

Let us consider a simple case of two-level hierarchy, with $q$ criteria compared at the upper level, and $m_{1}, m_{2}, \ldots, m_{q}$ alternatives compared within the $1^{\text {st }}, 2^{\text {nd }}$, etc., till the $q$-th criterion, respectively. Total of all the alternatives is a given constant $n$ :

$$
n=m_{1}+m_{2}+\ldots+m_{q} \text {. }
$$

The total of all paired combinations among the criteria and alternatives by the criteria equals:

$$
T=\frac{q(q-1)}{2}+\sum_{j=1}^{q} \frac{m_{j}\left(m_{j}-1\right)}{2} .
$$

Adding the restriction (1) yields the conditional objective:

$$
T=\frac{q(q-1)}{2}+\sum_{j=1}^{q} \frac{m_{j}\left(m_{j}-1\right)}{2}-\lambda\left(\sum_{j=1}^{q} m_{j}-n\right),
$$

where $\lambda$ is a Lagrange term. The quadratic items in (3) describe the convex functions, so the minimum by $m_{j}$ can be defined by the condition of each partial derivative equals zero:

$$
\frac{\partial T}{\partial m_{j}}=m_{j}-\frac{1}{2}-\lambda=0, \quad j=1,2, \ldots, q .
$$

Summing equations (5) by $j$ and dividing by $q$ yields:

$$
\lambda+\frac{1}{2}=\frac{1}{q} \sum_{j=1}^{q} m_{j}=\frac{n}{q} \equiv m,
$$

where $m$ denotes a mean value of the sizes of all groups of alternatives (1). Substituting (5) into equations (4) shows that

$$
m_{j}=m=n / q, \quad j=1,2, \ldots, q,
$$

so the minimum number of the pairs (2) can be reached with equal division of all $n$ alternatives by $m$ of them into each of $q$ criteria groups. Using (6) in (2) yields the total as follows:

$$
T=\frac{q(q-1)}{2}+q \frac{(n / q)(n / q-1)}{2}=\frac{q(q-1)}{2}+\frac{n}{2}\left(\frac{n}{q}-1\right)
$$

International Symposium of 
where the first item in sum corresponds to the number of combinations at the upper level of $q$ criteria, and the second item is the number of combinations in $q$ groups of $m$ alternatives in each at the lower level of the hierarchy.

The formula (7) expresses the total number of pair comparisons via a given constant $n$ of all alternatives and the unknown number $q$ of criteria. Minimizing the objective (7) yields:

$$
\frac{d T}{d q}=q-\frac{1}{2}-\frac{n^{2}}{2 q^{2}}=0, \quad \frac{d^{2} T}{d q^{2}}=1+\frac{n^{2}}{q^{3}}>0 .
$$

So the first order derivative corresponds to minimum and can be reduced to the equation:

$$
q^{3}-q^{2} / 2-n^{2} / 2=0 .
$$

This cubic equation has only one real root which can be simplified to the expression:

$$
q=2^{-1 / 3} n^{2 / 3}=0.79 n^{2 / 3} \text {. }
$$

Then for the optimum number (10) of criteria, the number of alternatives within each criterion can be estimated by (6) as:

$$
m=n / q=2^{1 / 3} n^{1 / 3}=1.26 n^{1 / 3} .
$$

Then the minimum number of total pair comparisons (7) can be reduced to the formula:

$$
T_{\min }=\frac{3}{2 \cdot 2^{2 / 3}} n^{4 / 3}=0.94 n^{4 / 3}
$$

so the rate $n^{4 / 3}$ of the pairs' number increase as $n$ grows is slightly higher than a linear one.

The total number of all pairs without reducing the number of needed comparisons can be estimated as number of combinations (1) from $n$ alternatives, $T_{\max }=n(n-1) / 2$, which is the quadratic function of $n$. The quotient of the minimum (12) to this maximum of the needed number of pairs can be presented as:

$$
\frac{T_{\min }}{T_{\max }} \approx \frac{0.94 n^{4 / 3}}{n(n-1) / 2} \approx 1.88 n^{-2 / 3} .
$$

With $n$ increasing this ratio is quickly diminishing, so the structuring of the alternatives into two hierarchy levels significantly reduces the needed work of data eliciting and processing.

\section{Data/Model Analysis}

For a given number of the alternatives $\mathrm{n}$ from 5 to 100 , the results of the hierarchy parameters' evaluation by the formulae (10)-(13) are presented in the table given in Appendix. Of course, we have to pick the values $\mathrm{q}$ and $\mathrm{m}$ as integers in a vicinity of the values shown in that table. For instance, with $n=53$ alternatives, the probable best value for the number of criteria should be about $\mathrm{q}=11$, by $\mathrm{m}=5$ items compared within each criterion. Then the total number of all pair comparisons can be reduced from the maximum value $T \max =1378$ to $T \min =156$, so almost by 9 times. More complicated hierarchies with three and more levels of criteria can be studied in a similar approach.

\section{Limitations}

In the described approach we can only obtain very rough estimates of the optimum hierarchy structures. More exact values can be found by the methods of integer quadratic programming and other techniques of integer optimization (Saaty, 1970).

\section{Conclusions}


ISAHP Article: Lipovetsky, Paper Proposal Submitted to the International Symposium of the Analytic Hierarchy Process 2014, Washington D.C., U.S.A.

A problem of finding an optimal structure of the hierarchy for a given number of alternatives is considered. The suggested technique is based on minimizing the objective of total pair comparisons across all the hierarchy levels. The obtained analytical and numerical results show how to choose the optimal structuring of the alternatives into groups by criteria. Such an approach can be extended to a many level hierarchy as well. This approach can significantly reduce the efforts in eliciting data and synthesizing the local preferences into global priorities, and be beneficial for practical managerial decision making in complex problems with numerous alternatives. Future developments should be based on more exact methods of optimization in integers (see Saaty, 1970).

\section{Key References}

Lipovetsky, S. (2006). Optimal Hierarchy Structures for Multi-Attribute-Criteria Decisions. Journal of Systems Science and Complexity,22, 228-242.

Lipovetsky, S. (2009). Global Priority Estimation in Multiperson Decision Making, Journal of Optimization Theory and Applications, 140, 77-91.

Saaty, T.L. (1970). Optimization in Integers and Related Extremal Problems, McGraw-Hill, New York.

Saaty, T.L., (1980). The Analytic Hierarchy Process. McGraw-Hill, New York.

Saaty, T.L. (1994). Fundamentals of Decision Making and Priority Theory with the Analytic Hierarchy Process. RWS Publications, Pittsburgh.

\section{Appendix. Two-Level Hierarchy Optimal Parameters.}

\begin{tabular}{|ccccc|ccccc|}
\hline \multicolumn{7}{c}{} & \multicolumn{7}{c}{$\boldsymbol{T m \boldsymbol { i }}$} & & & & & & \\
$\boldsymbol{n}$ & $\boldsymbol{m}$ & $\boldsymbol{q}$ & $\boldsymbol{n}$ & $\boldsymbol{T m a x}$ & $\boldsymbol{n}$ & $\boldsymbol{m}$ & $\boldsymbol{q}$ & $\boldsymbol{T m i n}$ & $\boldsymbol{T m a x}$ \\
\hline 5 & 2.2 & 2.3 & 4.4 & 10 & 53 & 4.7 & 11.2 & 156.0 & 1378 \\
6 & 2.3 & 2.6 & 6.0 & 15 & 54 & 4.8 & 11.3 & 160.2 & 1431 \\
7 & 2.4 & 2.9 & 7.7 & 21 & 55 & 4.8 & 11.5 & 164.4 & 1485 \\
8 & 2.5 & 3.2 & 9.5 & 28 & 56 & 4.8 & 11.6 & 168.6 & 1540 \\
9 & 2.6 & 3.4 & 11.5 & 36 & 57 & 4.8 & 11.8 & 172.9 & 1596 \\
10 & 2.7 & 3.7 & 13.5 & 45 & 58 & 4.9 & 11.9 & 177.2 & 1653 \\
11 & 2.8 & 3.9 & 15.7 & 55 & 59 & 4.9 & 12.0 & 181.5 & 1711 \\
12 & 2.9 & 4.2 & 17.9 & 66 & 60 & 4.9 & 12.2 & 185.9 & 1770 \\
13 & 3.0 & 4.4 & 20.2 & 78 & 61 & 5.0 & 12.3 & 190.3 & 1830 \\
14 & 3.0 & 4.6 & 22.6 & 91 & 62 & 5.0 & 12.4 & 194.7 & 1891 \\
15 & 3.1 & 4.8 & 25.0 & 105 & 63 & 5.0 & 12.6 & 199.1 & 1953 \\
16 & 3.2 & 5.0 & 27.6 & 120 & 64 & 5.0 & 12.7 & 203.6 & 2016 \\
17 & 3.2 & 5.2 & 30.2 & 136 & 65 & 5.1 & 12.8 & 208.0 & 2080 \\
18 & 3.3 & 5.5 & 32.9 & 153 & 66 & 5.1 & 13.0 & 212.6 & 2145 \\
19 & 3.4 & 5.7 & 35.6 & 171 & 67 & 5.1 & 13.1 & 217.1 & 2211
\end{tabular}


ISAHP Article: Lipovetsky, Paper Proposal Submitted to the International Symposium of the Analytic Hierarchy Process 2014, Washington D.C., U.S.A.

\begin{tabular}{|c|c|c|c|c|c|c|c|c|c|}
\hline 20 & 3.4 & 5.8 & 38.4 & 190 & 68 & 5.1 & 13.2 & 221.7 & 2278 \\
\hline 21 & 3.5 & 6.0 & 41.2 & 210 & 69 & 5.2 & 13.4 & 226.2 & 2346 \\
\hline 22 & 3.5 & 6.2 & 44.1 & 231 & 70 & 5.2 & 13.5 & 230.9 & 2415 \\
\hline 23 & 3.6 & 6.4 & 47.1 & 253 & 71 & 5.2 & 13.6 & 235.5 & 2485 \\
\hline 24 & 3.6 & 6.6 & 50.1 & 276 & 72 & 5.2 & 13.7 & 240.2 & 2556 \\
\hline 25 & 3.7 & 6.8 & 53.2 & 300 & 73 & 5.3 & 13.9 & 244.9 & 2628 \\
\hline 26 & 3.7 & 7.0 & 56.3 & 325 & 74 & 5.3 & 14.0 & 249.6 & 2701 \\
\hline 27 & 3.8 & 7.1 & 59.5 & 351 & 75 & 5.3 & 14.1 & 254.3 & 2775 \\
\hline 28 & 3.8 & 7.3 & 62.7 & 378 & 76 & 5.3 & 14.2 & 259.1 & 2850 \\
\hline 29 & 3.9 & 7.5 & 65.9 & 406 & 77 & 5.4 & 14.4 & 263.9 & 2926 \\
\hline 30 & 3.9 & 7.7 & 69.3 & 435 & 78 & 5.4 & 14.5 & 268.7 & 3003 \\
\hline 31 & 4.0 & 7.8 & 72.6 & 465 & 79 & 5.4 & 14.6 & 273.5 & 3081 \\
\hline 32 & 4.0 & 8.0 & 76.0 & 496 & 80 & 5.4 & 14.7 & 278.4 & 3160 \\
\hline 33 & 4.0 & 8.2 & 79.4 & 528 & 81 & 5.5 & 14.9 & 283.2 & 3240 \\
\hline 34 & 4.1 & 8.3 & 82.9 & 561 & 82 & 5.5 & 15.0 & 288.1 & 3321 \\
\hline 35 & 4.1 & 8.5 & 86.4 & 595 & 83 & 5.5 & 15.1 & 293.1 & 3403 \\
\hline 36 & 4.2 & 8.7 & 90.0 & 630 & 84 & 5.5 & 15.2 & 298.0 & 3486 \\
\hline 37 & 4.2 & 8.8 & 93.6 & 666 & 85 & 5.5 & 15.3 & 303.0 & 3570 \\
\hline 38 & 4.2 & 9.0 & 97.2 & 703 & 86 & 5.6 & 15.5 & 308.0 & 3655 \\
\hline 39 & 4.3 & 9.1 & 100.9 & 741 & 87 & 5.6 & 15.6 & 313.0 & 3741 \\
\hline 40 & 4.3 & 9.3 & 104.6 & 780 & 88 & 5.6 & 15.7 & 318.0 & 3828 \\
\hline 41 & 4.3 & 9.4 & 108.4 & 820 & 89 & 5.6 & 15.8 & 323.1 & 3916 \\
\hline 42 & 4.4 & 9.6 & 112.2 & 861 & 90 & 5.6 & 15.9 & 328.1 & 4005 \\
\hline 43 & 4.4 & 9.7 & 116.0 & 903 & 91 & 5.7 & 16.1 & 333.2 & 4095 \\
\hline 44 & 4.4 & 9.9 & 119.8 & 946 & 92 & 5.7 & 16.2 & 338.4 & 4186 \\
\hline 45 & 4.5 & 10.0 & 123.7 & 990 & 93 & 5.7 & 16.3 & 343.5 & 4278 \\
\hline 46 & 4.5 & 10.2 & 127.7 & 1035 & 94 & 5.7 & 16.4 & 348.7 & 4371 \\
\hline 47 & 4.5 & 10.3 & 131.6 & 1081 & 95 & 5.7 & 16.5 & 353.8 & 4465 \\
\hline 48 & 4.6 & 10.5 & 135.6 & 1128 & 96 & 5.8 & 16.6 & 359.0 & 4560 \\
\hline 49 & 4.6 & 10.6 & 139.6 & 1176 & 97 & 5.8 & 16.8 & 364.3 & 4656 \\
\hline 50 & 4.6 & 10.8 & 143.7 & 1225 & 98 & 5.8 & 16.9 & 369.5 & 4753 \\
\hline 51 & 4.7 & 10.9 & 147.8 & 1275 & 99 & 5.8 & 17.0 & 374.8 & 4851 \\
\hline 52 & 4.7 & 11.1 & 151.9 & 1326 & 100 & 5.8 & 17.1 & 380.1 & 4950 \\
\hline
\end{tabular}

